\title{
Improvement of an Octave Bandwidth Bowtie Antenna Design Based on the Analysis of a MIMO Efficiency Metric in Random-LOS
}

\author{
Sadegh Mansouri Moghaddam, Andrés Alayón Glazunov, Senior Member, IEEE, Per-Simon Kildal, Fellow, IEEE,
} Jian Yang, Senior Member, IEEE and Mattias Gustafsson

\begin{abstract}
A MIMO efficiency metric based on the probability of detection (PoD) of data bitstreams is used to improve the design of a self-grounded dual-polarized wideband bowtie antenna over an octave bandwidth. The analysis is based on the ideal digital threshold receiver model for the Zero-Forcing algorithm. The random line-of-sight (Random-LOS) channel model with two orthogonal linearly polarized waves coming from the same angleof-arrival is considered in the evaluation. The MIMO efficiency performance is presented along with the reflection coefficient, the directivity and the radiation patterns of the antennas. Corresponding spatial throughput coverage patterns based on 2-bitstream MIMO efficiency evaluated at the 95\% PoD level are also provided. The loss of orthogonality as well as the power imbalance of the 2-bitstreams has been identified as the main reasons to MIMO efficiency performance degradation in Random-LOS.
\end{abstract}

Index Terms-MIMO, Random-LOS, self-grounded bowtie, Probability of Detection (PoD), Zero-Forcing.

\section{INTRODUCTION}

As well-known, the multiple-input multiple-output (MIMO) data throughput can be maximized in terms of bitstream multiplexing or diversity by separating the antennas at least a half wavelength apart. However, this approach cannot be employed when the space is limited, e.g., in micro base stations. The compactness and high port-isolation constraints call for the use of polarization diversity [1], [2]. In most previous works, the performance of systems employing polarization-MIMO has been evaluated in multipath environments [3]. However, there might be a line-of-sight (LOS) component between the transmitter and the receiver with a very small diffuse scattering component. The orientation and the location of users with respect to the base station changes unpredictably [4]. This will result in a random variation of the angle-of-arrival (AoA) and the polarization of the LOS component that can be mitigated by polarization diversity [5].

The term Random-LOS has been introduced to describe the above channel in the limiting case [6]. This channel has

This work has been supported by two projects from Sweden's innovation agency VINNOVA, one within the VINN Excellence Center Chase at Chalmers and another via the program Innovative ICT 2013, and by internal support from Chalmers.

Sadegh Mansouri Moghaddam, Andrés Alayón Glazunov, Per-Simon Kildal (deceased) and Jian Yang are with the Department of Signals and Systems, Chalmers University of Technology, Gothenburg, Sweden (sadegh.mansouri@chalmers.se, andres.glazunov@chalmers.se and jian.yang@chalmers.se). Mattias Gustafsson is with Huawei Technologies Sweden AB.(Mattias.Gustafsson@huawei.com) been successfully employed to characterize MIMO-diversity and MIMO-multiplexing [7]. Recently, we discovered the performance degradation, in Random-LOS, of an earlier design of the self-grounded bowtie antenna presented in [8]. This earlier design was used as a 4-port antenna, so its poor 2-bitstream performance could not be detected. The 2-port antenna was realized by differentially exciting two opposite petals to produce two orthogonally polarized ports [9].

The novelty of this work is summarize next. We present a new improved design of the self-grounded bowtie antenna in a 2-port realization. We put emphasis on a novel system throughput performance characterization based on the analysis of a MIMO efficiency metric in Random-LOS. Also, we identify the polarization orthogonality and the power imbalance of the 2-bitstreams as the two main factors contributing to MIMO performance degradation in Random-LOS. The antenna achieves good MIMO efficiency over an octave bandwidth, i.e., $1.65-3.3 \mathrm{GHz}$, covering the target bandwidth $1.7-2.7 \mathrm{GHz}$.

Since the main focus is on the system performance characterization approach, results are presented based on simulations. The electromagnetic design of the antenna has been performed with the CST Microwave Studio. The far-field functions of the antennas were extracted to evaluate the 2-bitstream MIMO efficiency performance computed with a MATLAB-based simulation tool [10].

\section{SYSTEM PERFORMANCE CHARACTERIZATION}

According to the threshold receiver model [11], the average relative throughput can be interpreted as the probability of detection (PoD) of the data bitstreams. Since we consider the Random-LOS propagation environment, there can be maximum two bitstreams for co-located antennas corresponding to the two orthogonal polarizations. In this study, we specialize our analysis to linearly polarized antennas and impinging LOS field since in practical systems small terminal antennas are normally linearly polarized. Furthermore, we consider a wallmounted scenario for our bowties to simulate their deployment in micro base stations. Hence, the two random orthogonally polarized waves are incoming from the same AoA that is assumed to be uniformly distributed over the hemisphere. Each realization provides us with a $2 \times 2$ channel matrix which is post processed assuming a Zero-Forcing (ZF) receiver. The PoD for detection of 2 bitstreams is defined as the PoD of 
TABLE I. Dimensions of the bowtie antennas. The units of $\theta$ are given in $\left.{ }^{\circ}\right]$ and the rest are in $[\mathrm{mm}]$.

\begin{tabular}{c|cccccccc}
\hline & $\theta$ & $T_{w}$ & $l$ & $F_{d}$ & $P_{l}$ & $W_{g}$ & $W$ & $H$ \\
\hline \hline Earlier design & 45 & 17.5 & 5 & 11 & 80 & 100 & 111 & 29.5 \\
New design & 55 & 17.5 & 5 & 11 & 60 & 70 & 104 & 33.5 \\
\hline
\end{tabular}

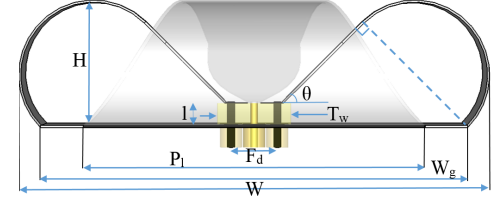

(a)

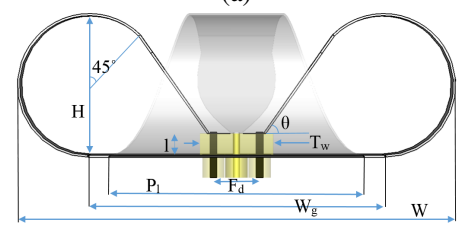

(c)

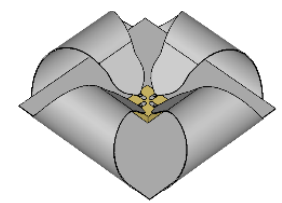

(b)

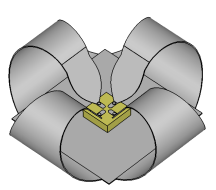

(d)
Fig. 1. Self-grounded dual-polarized wideband bowtie antenna. (a) Side view and (b) 3D view of an earlier design. (c) Side view and (d) 3D view of the new design.

detecting the weakest bitstream channel in each realization of the channel matrix.

We define the MIMO efficiency as in [12]

$$
\eta_{\mathrm{MIMO}}=\frac{\mathrm{PoD}_{\mathrm{ref}}^{-1}(0.95)}{\mathrm{PoD}^{-1}(0.95)}
$$

where $\operatorname{PoD}^{-1}$ is the inverse function of $\operatorname{PoD}\left(P_{\mathrm{av}} / P_{\mathrm{th}}\right)$, and $\operatorname{PoD}_{\text {ref }}\left(P_{\mathrm{av}} / P_{\mathrm{th}}\right)$ represents the probability of detection in the reference case. $P_{\mathrm{av}}$ and $P_{\mathrm{th}}$ are the average receive power and the power threshold of the receiver, respectively. The former is proportional to the transmit power, while the latter defines the receiver sensitivity. The reference here is a $100 \%$ efficient, 2-port antenna with perfectly orthogonally polarized far-field functions and with the equal gain over the hemisphere. We refer to this antennas as hemi-isotropic. In addition, the system performance can be characterized in terms of the MIMO throughput coverage pattern first introduced in [7]. The throughput coverage is understood here as relative throughput coverage and is denoted by $\operatorname{PoD}(\theta, \phi)$, where $\theta$ and $\phi$ are the AoA of the LOS orthogonally polarized waves impinging at the antenna, respectively.

\section{ANTENNA STRUCTURES AND PERFORMANCE COMPARISON}

\section{A. Antenna Geometry}

Fig. 1 shows a schematic representation of both the earlier design of the self-grounded dual-polarized bowtie antenna and the new design optimized in terms of MIMO efficiency. The dimensions of the antennas are listed in Table I.

The common features are the following: both antennas consist of 4 petals that rise from separate feed points at the surface of a Teflon block supporting the 4 feeding wires through the ground plane. These are connected to a PCB with a microstrip $180^{\circ}$-hybrid below the ground plane, working as a balun [13]. In the present simulations, the wires are

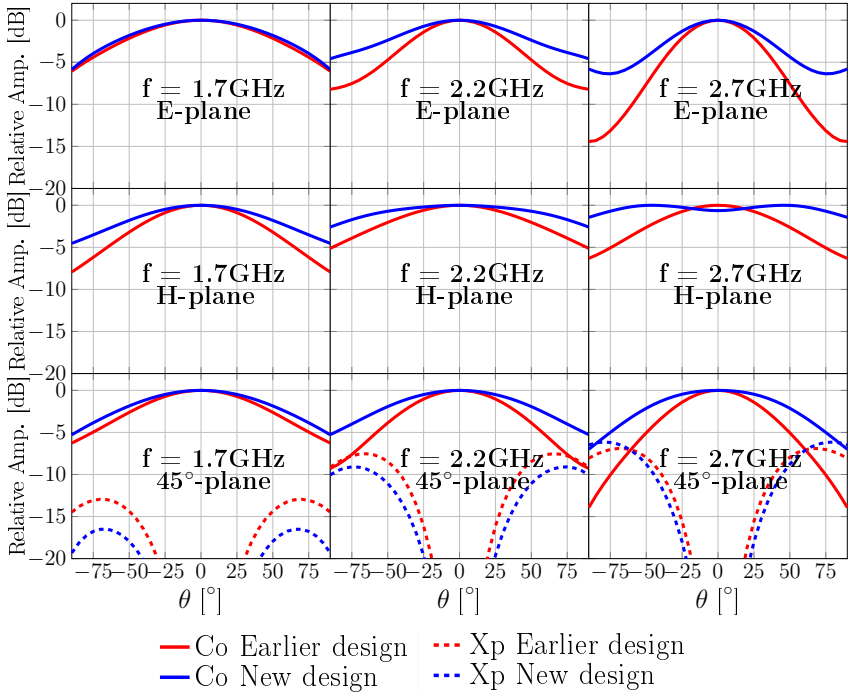

Fig. 2. Normalized co- and cross-polar radiation patterns of the simulated bowtie antenna designs in the E-plane (upper), H-plane (middle) and $45^{\circ}$ plane (lower), at $1.7 \mathrm{GHz}$ (left), $2.2 \mathrm{GHz}$ (middle) and $2.7 \mathrm{GHz}$ (right).

the extended center conductors of four $50 \Omega$ coaxial cables, which have their outer conductors connected to the ground plane. In order to study the performance of the bowties when realized as 2-port antennas with orthogonal polarizations, each of the two pairs of cables connected to opposite standing petals are differentially excited. Other common geometric features follow directly from Table I and Fig. 1.

The discerning features are the following: the size of the ground plane given by $W_{g}$ and the shape and size of the bowtie petals defined by $\theta, W, H$ and $P_{l}$ shown in Fig. 1 and Table I. The smaller ground plane in the new design was chosen since we aim to satisfy a maintained MIMO efficiency over a hemispherical coverage obtained with antenna patterns of wide enough beamwidths. The shape of the bowtie petal was changed in order to maintain the frequency bandwidth while reducing the size of the ground plane. In the earlier design, the petal's shape was obtained by bending over the half-perimeter of a virtual cylinder which diameter makes an angle equal to $90^{\circ}-\theta$ with respect to the ground plane as shown in Fig. 1(a). In this case, increasing $\theta$ results in a shorter length of petals coming straight out of the feed point. Thus, the optimization of the antenna in terms of far-field function, especially at higher frequencies, is restricted by the petal's geometry. To overcome these problems in the new design, the cylinder has been rotated to make a $90^{\circ}$ angle with respect to the ground plane as shown in Fig. 1(c). The bending is now over $5 / 8$ of the cylinder's perimeter. All the other parameters have been optimized in CST to obtain the required performance.

Another factor that will affect the MIMO throughput coverage of the antenna is the polarization orthogonality of the far-field function of the two ports over the hemisphere. In the earlier design, each differentially excited petal works more like a dipole over a ground plane. Hence, the far-field functions of two orthogonal petals will have almost parallel polarizations in the $45^{\circ}$-plane [14]. In the new design, by having the smaller ground plane and folding the petals at a greater angle $\theta$ than the earlier one, good orthogonality of the radiation pattern is 


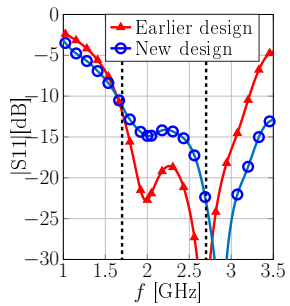

(a)

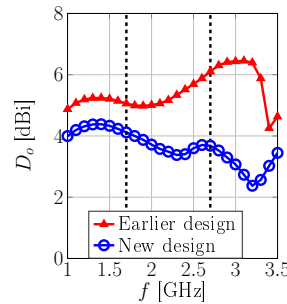

(b)

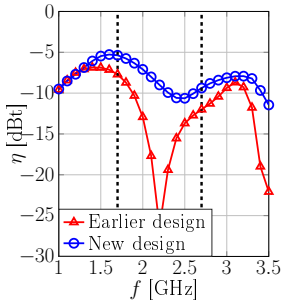

(c)
Fig. 3. Comparison of a) reflection coefficient and b) directivity of the two designs and c) the MIMO multiplexing efficiency in Random-LOS for hemispherical coverage vs. frequency

achieved in the $45^{\circ}$-plane too.

\section{B. Radiation pattern, reflection coefficient and directivity}

Fig. 2 shows the normalized co- and cross-polar radiation patterns of both bowties in three different planes, i.e., the E-plane, the H-plane and the $45^{\circ}$-plane. Both ports of the same bowtie have the same far-field function in their basic planes due to symmetry. Results are shown for three different frequencies. As can be seen, the radiation pattern is wider for the new design than for the earlier one, especially at higher frequencies. The cross-polar levels in the $45^{\circ}$-plane are almost the same. The cross-polar levels in the E- and H-planes are not visible since they are too low compared with the corresponding co-polar patterns.

The computed reflection coefficient and directivity are shown in Fig. 3(a) and (b), respectively. The coupling between the two orthogonally polarized differentially excited ports is very low and therefore it is not shown. It is worthwhile to note that for all the results presented here, port 2 was terminated in $50 \Omega$ when the performance on port 1 is computed. As can be seen, the reflection coefficient is below $-10 \mathrm{~dB}$ within the desired bandwidth, $1.7-2.7 \mathrm{GHz}$, for both designs. The corresponding directivity is within $5-6 \mathrm{dBi}$ and $3.5-4 \mathrm{dBi}$ for the earlier and the new designs, respectively.

\section{MIMO efficiency comparison}

The corresponding PoD curves for the two different antennas at three different frequencies are plotted in Fig. 4. The power level corresponding to $\mathrm{PoD}=0 \%$ is the maximum received power obtained from the $\mathrm{ZF}$ algorithm. This value, evaluated in $\mathrm{dB}$, equals the negative of the maximum realized gain of the antenna minus $3 \mathrm{~dB}$. The $3 \mathrm{~dB}$ arise from the facts that the bowtie antenna radiates orthogonally to its ground plane, and that the power of the incident wave is equally divided between the two bitstreams. The more vertical the PoD curve, the closer is the throughput coverage to the desired hemispherical coverage. This can be achieved by a $100 \%$ efficient 2-port antenna with perfectly orthogonally polarized far-field functions, both covering the whole hemisphere with the same gain, which we use as a reference. For example, as can be seen from Fig. 4(b), in order to detect 2 bitstreams at $2.2 \mathrm{GHz}$ for $95 \%$ of cases, i.e., for $\mathrm{PoD}=95 \%$, different amounts of transmit power are required depending on the antenna design. Indeed, for the earlier design, $26 \mathrm{~dB}$ more transmit power or lower receiver sensitivity is required as

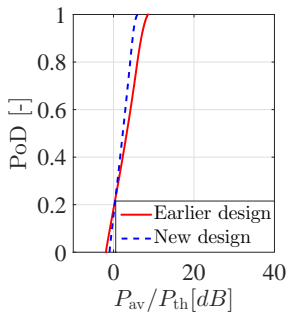

(a)

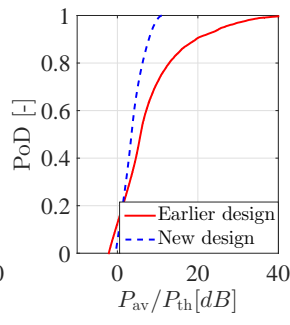

(b)

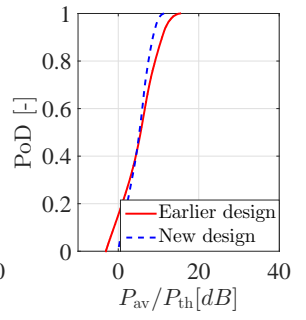

(c)
Fig. 4. Probability of Detection PoD of two bitstreams in Random-LOS with hemispherical coverage at a) $1.7 \mathrm{GHz}$, b) $2.2 \mathrm{GHz}$ and c) $2.7 \mathrm{GHz}$ for the two different designs.

compared to the reference case. On the other hand, the corresponding values for the new design is $9 \mathrm{~dB}$. Accordingly, $\eta_{\mathrm{MIMO}}=-26 \mathrm{~dB}$ and $\eta_{\mathrm{MIMO}}=-9 \mathrm{~dB}$ for the earlier and the new designs, respectively, i.e., a $17 \mathrm{~dB}$ improvement over the hemispherical angular coverage at $2.2 \mathrm{GHz}$.

Fig. 3(c) shows the MIMO efficiency (1) vs. frequency for both bowtie antenna designs. It is shown side by side with the reflection coefficient and the directivity for direct reference and comparison. $\eta_{\text {MIMO }}$ has been computed from PoD curves similar to Fig. 4 over a wide range of frequencies. As can be seen from Fig. 3(c), the MIMO efficiency of the new design considerably improves over the whole bandwidth as compared to the earlier design. For the new design, in order to achieve $95 \%$ throughput hemispherical coverage we need at most $10.5 \mathrm{~dB}$ more transmit power compared to the hemiisotropic reference, i.e., a $15.5 \mathrm{~dB}$ improvement over the earlier design.

\section{MIMO throughput coverage patterns}

Fig. 5 shows the hemispherical 2-bitstream MIMO throughput coverage pattern for the two bowtie antenna designs at three different frequencies. From Fig. 5(b) we see that $25 \mathrm{~dB}$ or more transmit power (or receiver sensitivity) is needed to detect both bitstreams in the $45^{\circ}$-plane as compared to the 2-port hemi-isotropic polarization-matched antenna reference. As shown in Fig. 5(e) this deficiency has been removed in the new design. The new antenna design provides a uniform coverage over the whole hemisphere and over the frequency band of interest. Fig. 6 and Fig. 7 show the polarization orthogonality $\left(1-I_{p}^{2}\right)$ and the amplitude imbalance $I_{a}^{2}$ factors, respectively. $I_{p}$ and $I_{a}$ were introduced in [14]. Comparing Fig. 5(a)-(f) with Fig. 6(a)-(f) and Fig. 7(a)-(f), we can readily see that, for the earlier design, the throughput coverage deeps coincide with low polarization orthogonality and high power imbalance. For example, the polarization non-orthogonality is the dominant factor which decreases the coverage in the $45^{\circ}$ plane at $2.2 \mathrm{GHz}$ while the power imbalance causes the bad performance in $\mathrm{E}$ and $\mathrm{H}$-plane at higher frequency. For the new design, the radiation patterns remain almost orthogonal and with better power balance over the whole hemisphere and over the whole bandwidth.

\section{Conclusions}

We have shown how to characterize and improve the design of a 2-port MIMO antenna in terms of 2-bitstream system 


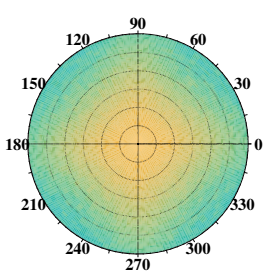

(a)

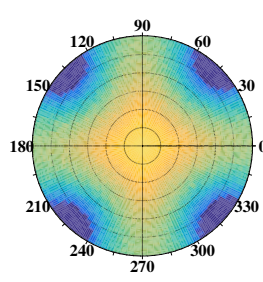

(b)

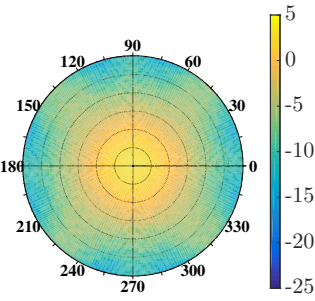

(c)

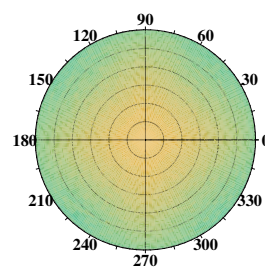

(d)

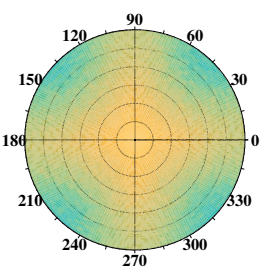

(e)

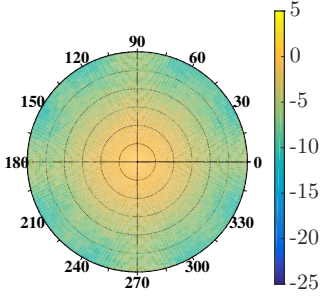

(f)

Fig. 5. 2-bitsream MIMO throughput coverage $\mathrm{PoD}(\theta, \phi)$, [dB], of the earlier design at (a) $1.7 \mathrm{GHz}$, (b) $2.2 \mathrm{GHz}$, (c) $2.7 \mathrm{GHz}$ and the new design at (d) $1.7 \mathrm{GHz}$, (e) $2.2 \mathrm{GHz}$, (f) $2.7 \mathrm{GHz}$.

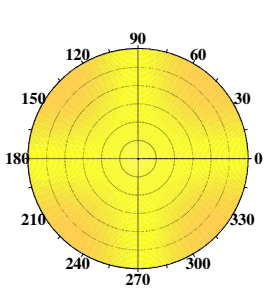

(a)

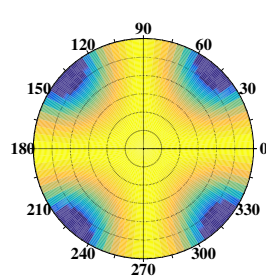

(b)

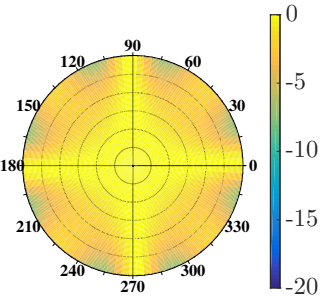

(c)

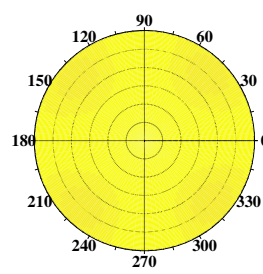

(d)

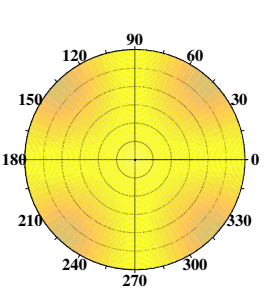

(e)

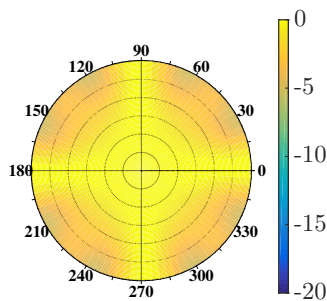

(f)

Fig. 6. Polarization orthogonality factor $\left(1-I_{p}^{2}(\theta, \phi)\right)$, [dB], of the earlier design at (a) $1.7 \mathrm{GHz}$, (b) $2.2 \mathrm{GHz}$, (c) $2.7 \mathrm{GHz}$ and the new design at (d) $1.7 \mathrm{GHz}$, (e) $2.2 \mathrm{GHz}$, (f) $2.7 \mathrm{GHz}$.

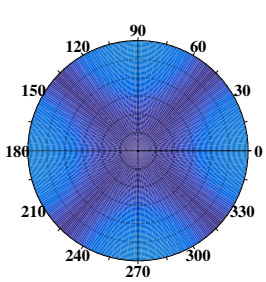

(a)

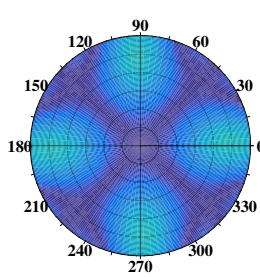

(b)

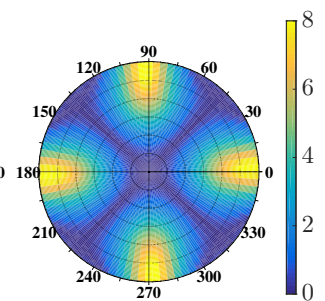

(c)

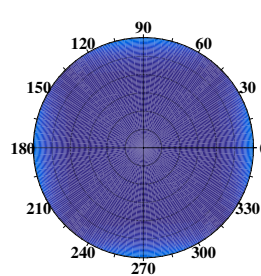

(d)

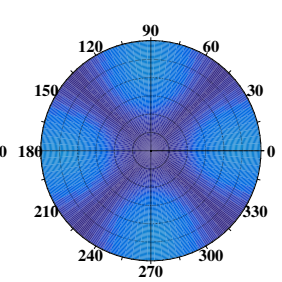

(e)

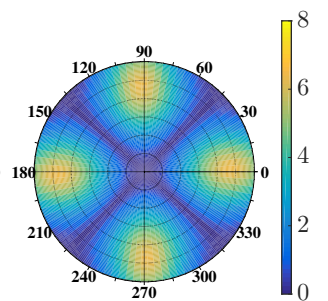

(f)

Fig. 7. Amplitude imbalance factor $I_{a}^{2}(\theta, \phi)$, [dB], of the earlier design at (a) $1.7 \mathrm{GHz}$, (b) $2.2 \mathrm{GHz}$, (c) $2.7 \mathrm{GHz}$ and the new design at (d) $1.7 \mathrm{GHz}$, (e) $2.2 \mathrm{GHz}$, (f) $2.7 \mathrm{GHz}$.

throughput performance in Random Line-Of-Sight. The new design has lower directivities, wider beamwidths and better orthogonality of the far-field functions corresponding to the two differentially excited ports of the antennas. The performance improvement is achieved over the target bandwidth of 1.7$2.7 \mathrm{GHz}$, but maintains its performance over an octave bandwidth, i.e., $1.65-3.3 \mathrm{GHz}$. The traditional cross-polar radiation pattern does not show a similar large improvement. Thus, the MIMO efficiency metric is an essential tool in the evaluation of the performance of an antenna design.

\section{REFERENCES}

[1] J. F. Valenzuela-Valdés, M. A. García-Fernández, A. M. MartínezGonzález, and D. A. Sánchez-Hernández, "Evaluation of true polarization diversity for MIMO systems," Antennas and Propagation, IEEE Transactions on, vol. 57, no. 9, pp. 2746-2755, 2009.

[2] P. G. Elliot, E. N. Rosario, R. J. Davis, and A. E. Rzhanov, "MIMO polarization diversity antenna with ultra-wide bandwidth and small size," in Phased Array Systems and Technology (ARRAY), 2010 IEEE International Symposium on. IEEE, 2010, pp. 559-566.

[3] N. K. Das, T. Inoue, T. Taniguchi, and Y. Karasawa, "An experiment on MIMO system having three orthogonal polarization diversity branches in multipath-rich environment," in Vehicular Technology Conference, 2004. VTC2004-Fall. 2004 IEEE 60th, vol. 2. IEEE, 2004, pp. 1528-1532.

[4] P. H. Lehne, K. Mahmood, A. A. Glazunov, P. Gronsund, and P. S. Kildal, "Measuring user-induced randomness to evaluate smart phone performance in real environments," in 2015 9th European Conference on Antennas and Propagation (EuCAP), May 2015, pp. 1-5.

[5] D. Cox, "Antenna diversity performance in mitigating the effects of portable radiotelephone orientation and multipath propagation," IEEE Trans. on Comm., vol. 31, no. 5, pp. 620-628, May 1983.
[6] P.-S. Kildal, "Rethinking the wireless channel for OTA testing and network optimization by including user statistics: RIMP, pure-LOS, throughput and detection probability," in ISAP 2013, Nanjing, 2013.

[7] P.-S. Kildal, X. Chen, M. Gustafsson, and Z. Shen, "MIMO characterization on system level of $5 \mathrm{G}$ microbase stations subject to randomness in LOS," Access, IEEE, vol. 2, pp. 1064-1077, 2014.

[8] H. Raza, A. Hussain, J. Yang, and P.-S. Kildal, "Wideband compact 4-port dual polarized self-grounded bowtie antenna," Antennas and Propagation, IEEE Tran., vol. 62, no. 9, pp. 4468-4473, 2014.

[9] S. M. Moghaddam, A. A. Glazunov, J. Yang, M. Gustafsson, and P. S. Kildal, "Comparison of 2-bitstream polarization-mimo performance of 2 and 4-port bowtie antennas for lte in random-los," in 2015 ISAP, Nov 2015 , pp. $1-4$.

[10] U. Carlberg, J. Carlsson, A. Hussain, and P.-S. Kildal, "Ray based multipath simulation tool for studying convergence and estimating ergodic capacity and diversity gain for antennas with given far-field functions," in ICECom, 2010 Conference Proceedings. IEEE, 2010, pp. 1-4 (Virmlab can be downloaded for free at www.kildal.se).

[11] P.-S. Kildal, A. Hussain, X. Chen, C. Orlenius, A. Skårbratt, J. Åsberg, T. Svensson, and T. Eriksson, "Threshold receiver model for throughput of wireless devices with MIMO and frequency diversity measured in reverberation chamber," Antennas and Wireless Propagation Letters, IEEE, vol. 10, pp. 1201-1204, 2011.

[12] A. Hussain, P. S. Kildal, and A. A. Glazunov, "Interpreting the total isotropic sensitivity and diversity gain of lte-enabled wireless devices from over-the-air throughput measurements in reverberation chambers," IEEE Access, vol. 3, pp. 131-145, 2015.

[13] S. Mansouri, J. Kvarnstrand, A. A. Glazunov, J. Yang, and P.-S. Kildal, "Double Broadband Balun Structure Using CRLH TL for Differential Excitation of Dual-Polarized Self-Grounded Bow-Tie Antenna," Session 3A12 SC4: Antenna-Channel Interactions and Wireless Propagation Channels, p. 1403, 2015.

[14] A. Razavi, A. A. Glazunov, P. S. Kildal, and J. Yang, "Investigation of polarization deficiencies in simo systems in random-los propagation channels," in 2015 ISAP, Nov 2015, pp. 1-3. 